\title{
Neuropsychological profile in a specific cohort of HIV patients infected postnatally: a cross-sectional study
}

This article was published in the following Dove Press journal:

Neurobehavioral HIV Medicine

9 October 2015

Number of times this article has been viewed

\author{
Silvia Riva ${ }^{1,2}$ \\ Ilaria Cutical \\ Gabriella Pravettoni',3 \\ 'Department of Health Sciences, \\ University of Milan, ${ }^{2}$ Department \\ of Neuroscience, IRCCS, Istituto \\ di Ricerche Farmacologiche "Mario \\ Negri", 'European Institute of \\ Oncology, Milan, Italy
}

\begin{abstract}
HIV-associated neurocognitive disorders (HANDs) are one of the most important complications of HIV infection reported in the current literature. Although HANDs have been closely studied in vertically infected HIV populations or in specific subgroups such as drug abusers or homosexuals, they have been completely understudied in hemophilia patients with HIV, infected through transfusions postnatally. For this reason, it seemed interesting to evaluate the presence of HAND in this specific population. The aim of this work is to present a study protocol aimed at assessing the neuropsychological profile of HIV+ hemophilia patients compared with that of HIV- hemophilia patients. This is the first European study to evaluate the neuropsychological profile of HIV+ adult hemophiliacs prospectively.
\end{abstract}

Keywords: neuropsychological profile, neuropsychology, HIV, cognitive disorders, hemophilia

\section{Background}

Human immunodeficiency virus (HIV) is highly neurovirulent. ${ }^{1}$ Prior to widespread availability of highly active antiretroviral therapy (HAART), the prevalence of HIVassociated cognitive impairment has been estimated to lie between $6 \%$ and $30 \%$, and this impairment could progress quickly to dementia or death. ${ }^{2-4}$ The introduction of HAART in 1996 has resulted in the suppression of the systemic HIV-1 viral load and improvements in survival for patients with HIV infection. ${ }^{5,6}$ Since 2009 , the term combination antiretroviral therapy (cART) has largely supplanted the more commonly known HAART, particularly among researchers and clinicians. It employs the use of three or more antiretroviral drugs, either taken individually or in fixed-dose combinations. Antiretroviral therapy (HAART/cART) has also been associated with an improvement in cognitive performance ${ }^{6}$ and a decreased incidence of HIV dementia. ${ }^{2,7}$ With treatment, the frequency of dementia has been attenuated, but it still remains uncertain whether the frequency of specific cognitive impairments in HIV infection has changed. HIV-associated neurocognitive disorders (HANDs) have not been yet eliminated, and they represent one of the most important complications of HIV infections. ${ }^{2,8,9}$ They are the result of neural damage caused by HIV replication and immune activation ${ }^{10}$ and are generally divided into three categories: 1) asymptomatic neurocognitive impairment, 2) mild neurocognitive disorder (MND), and 3) HIV-associated dementia. ${ }^{11}$ Asymptomatic neurocognitive impairment is detected when neuropsychological tests show HIVassociated impairments, but routine and daily functioning is not compromised. MND is detected when neuropsychological tests show HIV-associated impairments and mild impact in routine and daily functioning. HIV-associated dementia is detected when neuropsychological tests show important HIV-associated impairments and a severe
Correspondence: Silvia Riva

Department of Health Sciences,

University of Milan, Via A Di Rudini 8 ,

20142 Milan, Italy

Tel +39025032 I240

Email silvia.rival@unimi.it (c) (1) (5) 2015 Riva et al. This work is published by Dove Medical Press Limited, and licensed under Creative Commons Attribution - Non Commercial (unported, v3.0) License. The full terms of the License are available at http://creativecommons.org/licenses/by-nc/3.0/. Non-commercial uses of the work are permitted without any further (ical Press Limited, provided the work is properly attributed. Permissions beyond the scope of the License are administered by Dove Medical Press Limited. Information on how to request permission may be found at: http://www.dovepress.com/permissions.php 
impact on daily functioning. This impairment significantly limits one's ability to function day-to-day at work, home, and during social activities. MND appears to be the most common type of HAND. ${ }^{11}$ Despite its name, even mild cognitive problems can interfere with everyday functioning and reduce the quality of life. Estimates indicate that as many as $50 \%$ of HIV+ individuals display some degree of cognitive impairment ${ }^{10}$ when impairment is derived from comparisons with normative performance standards. ${ }^{6,10}$

While HAND are well studied in HIV-seropositive $(\mathrm{HIV}+)$ patients infected perinatally, these disorders remain comparatively understudied in the HIV+ population infected postnatally, suggesting that research should address the potential for neurocognitive complications in this cohort of patients. Indeed, neuropsychological impairments have been mainly studied in HIV-seropositive $(\mathrm{HIV}+)$ patients infected postnatally during the earliest period of life of the child (eg, through breast milk) or in specific cohorts of adults such as homosexuals and drug users, but much less is known regarding individuals infected postnatally. ${ }^{12}$ As revealed in the literature, in the cohort of homosexuals and drug users, patients show different neuropsychological impairments, but these profiles are difficult to describe because they are often complicated by a myriad of confounding factors, which may be present in these patients, such as the abuse of substances (eg, alcohol, cocaine, and opiates), ${ }^{13,14}$ emotional disorders (eg, anxiety and depression), ${ }^{5}$ and different risk

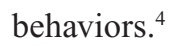

Furthermore, studies on HIV+ individuals infected postnatally, as is the case with hemophilia patients, are quite rare. This group of patients is quite distinct from other cohorts, the children infected postnatally through transfusions have a different path of the infection than those who were infected perinatally, ${ }^{15-17}$ and they generally do not present certain psychosocial vulnerabilities, which are sometimes related to perinatal infection and which may impact as covariate variables. Some such factors are, for instance, the severe illness or death of a parent due to AIDS or a lower socioeconomic status. Finally, the growth of patients infected postnatally may be different from the growth of patients infected perinatally because of the differences in the rate of brain growth; for instance, Rubin et a ${ }^{18}$ found different patterns of neurobehavioral development depending on age of infection, and Brouwers et $\mathrm{al}^{19}$ found differences in structural brain abnormalities between perinatally and transfusioninfected children.

The majority of individuals infected postnatally are hemophiliac patients. Due to the fact that HIV was not well known before 1985, many hemophilia (A/B) patients became silently infected through contaminated blood infusion and hence were at risk of developing AIDS. ${ }^{20}$ Patients with hemophilia are treated with injections of whole blood or other processed blood derivatives. Repetition of this treatment in the past exposed the hemophiliacs to the risk of bloodborne infections as HIV. ${ }^{21}$ Today, it is estimated that $60 \%-70 \%$ of people with hemophilia were infected with HIV in this way between 1979 and $1985 .^{22}$

Hemophilia is a rare coagulation disorder in which a crucial clotting factor in blood is missing either partly or completely. ${ }^{23-26}$ Being a sex chromosome-gene-related bleeding disorder, it occurs primarily in the male population. There are two main types of hemophilia. In the more common hemophilia A (eight of ten), patients are missing or have low levels of clotting factor VIII, whereas in hemophilia B patients are missing or have low levels of clotting factor IX. ${ }^{27}$ Most of the literature on HIV and hemophilia is derived from the Hemophilia Growth and Development Study (HGDS), ${ }^{28}$ which studied the longitudinal effect of the HIV infection on adolescents. The main publications of HGDS, for example, Sirois et al, ${ }^{29}$ Whitt et al, ${ }^{30}$ and Loveland et al, ${ }^{17}$ revealed that most of the asymptomatic cohort of HIV+ children and adolescents did not vary considerably from HIV- hemophilic controls on several neurocognitive tests at baseline. However, as shown in follow-up data of HGDS patients by Tombaugh et $\mathrm{al}^{31}$ and by Loveland et $\mathrm{al}^{20}$ over a period of 5 years, the majority of these young patients showed an important regression in neuropsychological performance, which was highly correlated to declines in immune functioning status. This regression, however, was not clearly associated with HIV infection, but it was associated with socioeducational variables such as school absenteeism and poorer academic achievement. Although the HGDS represents an important study in pediatric population, it is a quite dated study, which began before the introduction of HAART. Moreover, studies are totally underrepresented in the adult population, with mixed and confusing results. ${ }^{32}$ The only two exceptions from literature data found no significant differences at the MiniMental State Examination between patients with hemophiliac HIV and healthy controls; $;^{33,34}$ the only significant alterations in patients with hemophiliac HIV were found in patients suffering from spontaneous brain hemorrhage (microbleeds). Studies on adult hemophiliacs HIV+ are also novel because the survival rate of such patients has increased over recent decades as a result of the development of new therapies.

Based on these considerations, the present study protocol aims to evaluate the presence of HAND in HIV+ adult 
hemophiliacs in comparison with a control group of HIVhemophiliacs by administering a full neuropsychological battery.

\section{Objectives}

The primary objective of this study is to compare the neuropsychological profiles of patients with both hemophilia and HIV versus the profiles of patients with hemophilia who are not HIV infected.

The secondary objective is to evaluate the influence of age and other sociodemographic data (education and working status) in the cognitive profile of both groups.

The influence of sociodemographic variables has been extensively investigated in the HIV population; however, it has been underestimated and not evaluated in the specific group of HIV+ adult hemophiliac patients.

\section{Design}

The study will apply the STROBE (STrengthening the Reporting of OBservational studies in Epidemiology) procedures for observational studies. The STROBE checklist criteria for cohort, case-control, and cross-sectional studies will be fulfilled. ${ }^{35}$ This is an observational, cross-sectional, prospective study to be conducted in three hemophilia centers: Milan, Naples, and Rome.

The study will last for 12 months, and it will involve 45 patients with a ratio of 1:2 (15 HIV+ hemophiliacs and 30 HIV- hemophiliacs).

The group of HIV+ hemophiliacs will have the following characteristics:

- Age $\geq 30$ years

- Currently in cART regimens (including nucleotide reverse transcriptase inhibitors, nonnucleotide reverse transcriptase inhibitors, protease inhibitors, or integrase inhibitors)

- Diagnosis of hemophilia A or hemophilia B or von Willebrand disease

- Hemophilia diagnosis with any type of severity (severe hemophlia, Factor VIII [FVIII] level <1\%; moderate hemophilia, FVIII level ranging from 1\%-5\%; mild hemophilia, FVIII level ranging from 6\%-30\%)

- Diagnosis of HIV.

The control group will have the same characteristics, except for the diagnosis of HIV.

The criterion of age ( $>30$ years) was chosen based on the fact that patients were particularly exposed to the infection during the period 1979-1984 when the sources of FVIII level were contaminated with HIV. ${ }^{36}$
The protocol will exclude patients presenting the following characteristics:

- Presence of central neurological disorders and/or severe psychiatric disorders (diagnosed before/after HIV) that limit the execution of test assessment

- Presence of acute internal diseases

- Abuse of recreational drugs or alcohol interfering with everyday life in the opinion of the physician

- Concomitant use of antipsychotic treatment (eg, major tranquilizers) interfering with everyday life in the opinion of the physician.

\section{Psychological assessment Neuropsychological evaluation}

Participant (HIV+ and HIV-) hemophiliacs will complete the Italian Brief Neuropsychological Exam, ${ }^{37}$ including the measurement of six major cognitive domains. In this battery, standardize tests are included. The battery contains the most important standardized test for cognitive functions and is widely used in the Italian context. ${ }^{38,39}$ Attention will be measured by Trail Making Test, which is a neuropsychological test of visual attention and task switching. ${ }^{37}$ Working memory will be detected with the digit-span memory test using the LetterNumber Sequencing of the Wechsler Adult Intelligence Scale, third edition (WAIS-III), ${ }^{40}$ while phonemic memory will be assessed by the Verbal Fluency test. ${ }^{41}$ Abstraction, that is the ability of logical reasoning and generalization of concepts, will be measured by the Italian Test of abstraction of the Brief Neuropsychological Exam. ${ }^{37}$ Language will be measured with the short version of the Token Test, which is a valuable measure for assessing receptive language. ${ }^{42}$ Executive functions will be assessed with the Clock-Drawing Test. ${ }^{43}$ Finally, visual recognition will be detected through the Rey Tangled Lines Test. ${ }^{44}$

\section{Evaluation of daily life activities}

All patients will undergo a specific ad hoc interview for deterioration in daily living activities.

\section{Study procedures Schedule of appointments}

Regular appointments are expected to be performed at the three Hemophilia Centers, starting with the appointment in which written consent of the patient to data collection is obtained.

The patient identifier will consist of two parts, by which the patients will be uniquely identified:

- Center number (two digits)

- Patient identification number within center (two digits). 
A participating list will be provided for each center. For each specific center number, each enrolled patient will be consecutively documented.

\section{Statistical analysis}

This is a noninterventional study, and the statistical approach is exploratory in nature. With the $\alpha$ error of $5 \%$, and the power $(1-\beta)$ of $80 \%$ assuming a prevalence of impairment of $50 \%$ in hemophilia HIV cases and of $10 \%$ in the control group (patients with hemophilia HIV), the sample size estimated to conduct the study is, at least, 15 cases and 30 controls with a case:control ratio of 1:2. In order to avoid bias in the inclusion phase, a complete list of the patients will be collected, and they will be randomly chosen by using a random number generator.

Non-HIV patients will be matched with HIV patients by age ( \pm 5 years). The presence of HAND will be defined according to the cutoff normal value limits for each test, based on the internal control group (non-HIV hemophilic patients). HAND scores (for each test and as a global score) will be measured according to a standardized method as mild, moderate, and severe impairment.

Analyses will be performed using SPSS Version 21 statistical software (StataCorp LP, College Station, TX, USA). Alpha will be set at 0.05 , two tailed. Sociodemographic and clinical data (age, job status, and education) will be descriptively analyzed by means of frequency, median, mean, standard deviation, range, and confidence intervals. Differences in demographic variables between HIV groups (HIV+ and HIV-) will be examined using analysis of variance for continuous variables and Pearson's $\chi^{2}$ tests or Fisher's exact test for categorical variables. Years of education, job status, and age will be included as covariates in the analyses of neurocognitive data. The aging effect is modeled using both dichotomous baseline age groups (young, <45 years; old, $\geq 45$ years) and continuous age.

We are also interested in analyzing effect sizes. In order to calculate Cohen's $d$, the participants will be split into four groups: younger (age $<55$ years) HIV-, older (age 55 years and above) HIV-, younger HIV+, and older HIV+. Following these analyses, participants' scores will be converted into T-scores corrected for age, education, and job status to compare scores to established norms.

Raw scores for the individual cognitive tests will be converted to population-based $z$ scores (ie, individual score minus the group mean, divided by the group standard deviation) using raw score distributions from the clinical sample (ie, older HIV-infected sample) for standardization and comparison purposes.

\section{Ethical conduct and good clinical practice}

This study will be conducted in accordance with national laws. Ethics committees (ECs) and national agencies will be informed and their approval sought. Data protection regulations will be strictly adhered to at all times (eg, Directive 95/46/EC). This study will be carried out completely independent of any medicinal treatment recommendations. Patients will be assessed and treated according to the individual decision of the attending physician. The study will not provide any recommendations with regard to diagnostic procedures or dosages, time of intake, or the strength of any medication. All decisions are to be made by the physician in the best interest of the patient's care.

\section{Ethics committee and authority}

Before the study begins, the noninterventional plan, written data protection form(s), and/or other appropriate documents will be submitted to the EC, in accordance with local legal requirements. The patients will be provided with appropriate forms for obtaining written consent for data collection. In the case of minors, a written consent for the collection of data from the respective patient must also be obtained from the patient's parents or a legally authorized representative, as applicable, after adequate explanation of the purposes of this observational study and the objectives of data collection.

\section{Conclusion}

The last 2 decades have borne witness to dramatic improvements in the availability and quality of treatment for persons with HIV. Accordingly, a considerable number of these patients now live longer. In the context of hemophilia, the introduction of virus-inactivated plasma-derived coagulation factors and recombinant products has revolutionized the care of people with hemophilia, improving their health-related quality of life and life expectancy. However, living with two severe conditions such as HIV and hemophilia is challenging. As with any medical condition, it is very important to evaluate the psychological and cognitive processes related to multiple diseases and multiple treatments. ${ }^{45-49}$ This study is unique in the field of hemophilia, and it will offer an in-depth evaluation of the neuropsychological profile and a psychoemotional assessment of this particular cohort of patients with HIV. 


\section{Acknowledgment}

The first author acknowledges support from the Novo Nordisk grant "Changing Possibilities in Hemophilia ${ }^{\circledR ”}$ for the opportunity provided to conduct this study.

\section{Disclosure}

The authors report no conflicts of interest in this work.

\section{References}

1. Reger M, Welsh R, Razani J, Martin DJ, Boone KB. A meta-analysis of the neuropsychological sequelae of HIV infection. $J$ Int Neuropsychol Soc. 2002;8:410-424.

2. Antinori A, Arendt G, Becker JT, et al. Updated research nosology for HIV-associated neurocognitive disorders. Neurology. 2007;69: 1789-1799.

3. Heaton RK, Clifford DB, Franklin DR Jr, et al. HIV associated neurocognitive disorders persist in the era of potent antiretroviral therapy: CHARTER study. Neurology. 2010;75:2087-2096.

4. De Ronchi D, Faranca I, Berardi D, et al. Risk factors for cognitive impairment in HIV-1-Infected persons with different risk behaviours. Arch Neurol. 2002;59:812-818.

5. Byrd DA, Robinson-Papp J, Rivera Mindt R, et al. Isolating cognitive and neurologic HIV effects in substance-dependent, confounded cohorts: a pilot study. J Int Neuropsychol Soc. 2013;19:463-473.

6. Heaton RK, Franklin DR, Ellis RJ, et al. HIV-associated neurocognitive disorders before and during the era of combination antiretroviral therapy: differences in rates, nature, and predictors. J Neurovirol. 2011;17:3-16.

7. Al-Khindi T, Zakzanis KK, van Gorp WG. Does antiretroviral therapy improve HIV-associated cognitive impairment? A quantitative review of the literature. J Int Neuropsychol Soc. 2011;17:956-969.

8. Doyle KL, Loft S, Morgan EE, et al. Prospective memory in HIVassociated neurocognitive disorders (HAND): the neuropsychological dynamics of time monitoring. J Clin Exp Neuropsychol. 2013;35: 359-372.

9. Ellis RJ, Badiee J, Vaida F, et al. CD4 nadir is a predictor of HIV neurocognitive impairment in the era of combination antiretroviral therapy. AIDS. 2011;25:1747-1751.

10. Heaton RK, Franklin DR, Ellis RJ, et al; CHARTER Group, HNRC Group. HIV-associated neurocognitive disorders before and during the era of combination antiretroviral therapy: differences in rates, nature, and predictors. J Neurovirol. 2011;17:3-16.

11. Iudicello JE, Woods SP, Weber E, Dawson MS, Scott JC; The HIV Neurobehavioral Research Center (HNRC) Group. Cognitive mechanisms of switching in HIV-associated category fluency deficits. $J$ Clin Exp Neuropsychol. 2008;30:797-804.

12. Riva S, Cutica I, Pravettoni G. Is there evidence for neurocognitive dysfunctions in patients with postnatal HIV infection? A review on the cohort of hemophilia patients. Front Hum Neurosci. 2014;8:470.

13. Büttner A. Review: the neuropathology of drug abuse. Neuropathol Appl Neurobiol. 2011;37:118-134.

14. Lundqvist T. Imaging cognitive deficits in drug abuse. Curr Top Behav Neurosci. 2010;3:247-275.

15. Cohen SE, Mundy T, Karassik B, Lieb L, Ludwig DD, Ward J. Neuropsychological functioning in human immunodeficiency virus type 1 seropositive children infected through neonatal blood transfusion. Pediatrics. 1991;88:58-68.

16. Loveland KA, Stehbens J, Contant C, et al. Hemophilia growth and development study: baseline neurodevelopmental findings. J Pediatr Psychol. 1994;19:223-239.

17. Loveland KA, Stehbens JA, Mahoney EM, et al. Declining immune function in children and adolescents with hemophilia and HIV infection: effects on neuropsychological performance. J Pediatr Psychol. 2000;25: 309-322.
18. Rubin SA, Bautista JR, Moran TH, Schwartz GJ, Carbone KM. Viral teratogenesis: brain developmental damage associated with maturation state at time of infection. Brain Res Dev Brain Res. 1999;112:237-244.

19. Brouwers P, De Carli C, Civitello L, Moss H, Wolters P, Pizzo P. Correlation between computed tomographic brain scan abnormalities and neuropsychological function in children with symptomatic human immunodeficiency virus disease. Arch Neurol. 1995;52:39-44.

20. Franchini M, Mannucci PM. Past, present and future of hemophilia: a narrative review. Orphanet J Rare Dis. 2012;2(7):24.

21. Franchini M, Coppola A, Rocino A, Zanon E, Morfini M; The Italian Association of Hemophilia Centers (AICE) Working Group. Perceived challenges and attitudes to regimen and product selection from Italian hemophilia treaters: the 2013 AICE survey. Haemophilia. 2014;20: $128-135$.

22. Mannucci PM. Hemophilia: treatment options on the twenty-first century. J Thromb Haemost. 2005;1:1349-1355.

23. Krasuska M, Riva S, Fava L, von Mackensen S, Bullinger M. Using the international classification of functioning, disability and health in chronic health conditions: the example of young people with hemophilia. Am J Phys Med Rehabil. 2012;91:S74-S83.

24. Lessinger C, Gringeri A, Berntorp E, et al. Health-related quality of life in patients with hemophilia a and inhibitors on prophylaxis: results from the Pro-FEIBA study. Haemophilia. 2013;19(5):736-743.

25. Mangiafico L, Perja M, Fusco F, Riva S, Mago D, Gringeri A. Safety and effectiveness of raltegravir in patients with haemophilia and anti-HIV multidrug resistance. Haemophilia. 2011;18(1):108-111.

26. Muça-Perja M, Riva S, Grochowska B, Mangiafico L, Mago D, Gringeri A. Ultrasonography of haemophilic arthropathy. Haemophilia. 2011;18(3):364-368.

27. Santagostino E, Mannucci PM. Guidelines for replacement treatment of hemophilia and inherited coagulation disorders in Italy. Haemophilia. 2000;6:1-10.

28. Hilgartner MW, Donfield SM, Willoughby A, et al. Hemophilia growth and development study: design, methods, and entry data. $J$ Pediatr Hematol Oncol. 1993;15:208-218.

29. Sirois PA, Usner DW, Hill SD, et al. Hemophilia growth and development study: relationships between neuropsychological, neurological, and MRI findings at baseline. J Pediatr Psychol. 1998;23:45-56.

30. Whitt JK, Hooper SR, Tennison MB, et al. Neuropsychologic functioning of human immunodeficiency virus-infected children with hemophilia. J Pediatr. 1993;122:52-59.

31. Tombaugh NS, Mahoney EM, Sirois PA, et al. HIV-associated changes in adaptive, emotional, and behavioral functioning in children and adolescents with hemophilia: results from the hemophilia growth and development study. J Pediatr Psychol. 2000;25:545-556.

32. Riedel RR, Helmstaedter C, Bülau P, et al. Early signs of cognitive deficits among human immunodeficiency virus-positive hemophiliacs. Acta Psychiatr Scand. 1992;85:321-326.

33. Siboni SM, Mannucci PM, Gringeri A, et al. Health status and quality of life of elderly persons with severe hemophilia born before the advent of modern replacement therapy. J Thromb Haemost. 2009;7:780-786.

34. Zanon E, Manara R, Milan M, et al. Cognitive dysfunctions and cerebral microbleeds in adult patients with haemophilia A: a clinical and MRI pilot-study. Thromb Res. 2014;134:851-855.

35. von Elm E, Altman DG, Egger M, et al; STROBE Initiative. The Strengthening the Reporting of Observational Studies in Epidemiology (STROBE) statement: guidelines for reporting observational studies. Lancet. 2007;370(9596):1453-1457.

36. Jewell NP. Quantifying the source of infection for HIV-infected hemophiliacs in the UK from 1979 to 1984. Stat Med. 2009;28(10): 1464-1472.

37. Mondini S, Mapelli D, Vestri A, Arcara G, Bisiacchi PS. Esame Neuropsicologico Breve. Torino: Cortina Raffaello; 2003.

38. Bisiacchi PS, Suppiej A, Laverda A. Neuropsychological evaluation of neurologically asymptomatic HIV infected children. Brain Cogn. 2000;43:49-52. 
39. Amendola G, Caprioglio S, Mantia E, Spagna S, Zoppi M, Montobbio G. Studio descrittivo delle evidenze neuropsicologiche in un gruppo di pazienti HIV positivi afferenti al reparto malattie infettive dell'ospedale di Alessandria. [A descriptive study of neuropsychological findings in a group of positive HIV patients hospitalized in an infectious diseases department at the hospital of Alessandria]. Work Pap Public Health. 2012;1:1-21. Italian.

40. Wechsler S. The Semantic Basis of Argument Structure. Stanford, NY: Cambridge University Press; CSLI Publications (Center for the Study of Language and Information); 1995.

41. Lezak MD. Neuropsychological Assessment. Oxford: Oxford University Press; 1995.

42. Spellacy FJ, Spreen O. A short form of the token test. Cortex. 1969;5: 390-397.

43. Bisiacchi PS, Borella E, Bergamaschi S, Carretti B, Mondini S. Interplay between memory and executive functions in normal and pathological aging. J Clin Exp Neuropsychol. 2008;30(6):723-733.
44. Rey A. L'Examen clinique en psycologie. [The Clinical Exam in Psychology]. Paris: Presses Universitaires de France; 1964. French.

45. Riva S, Monti M, Iannello P, Pravettoni G, Schulz PJ, Antonietti A. A preliminary mixed-method investigation of trust and hidden signals in medical consultations. PLoS One. 2014;9(3):e90941.

46. Schulz PJ, Hartung U, Riva S. Causes, coping, and culture: a comparative survey study on representation of back pain in three Swiss language regions. PLoS One. 2013;8(11):e78029.

47. Riva S, Monti M, Iannello P, Antonietti A. The representation of risk in routine medical experience: what actions for contemporary health policy? PLoS One. 2012;7(11):e48297.

48. Riva S, Camerini AL, Allam A, Schulz PJ. Interactive sections of an internet-based intervention increase empowerment of chronic back pain patients: randomized controlled trial. J Med Internet Res. 2014; 16(8):e180.

49. Baldi P, Iannello P, Riva S, Antonietti A. Cognitive reflection and socially biased decisions. Stud Psychol. 2013;55:265-271.
Neurobehavioral HIV Medicine

\section{Publish your work in this journal}

Neurobehavioral HIV Medicine is an international, peer-reviewed, open access journal focusing on advances in research in HIV/ AIDS, with specific reference to the neurological, psychiatric and behavioral consequences of the disease, concomitant infections and specific antiretroviral therapy. The manuscript

\section{Dovepress}

management system is completely online and includes a very quick and fair peer-review system, which is all easy to use Visit http://www.dovepress.com/testimonials.php to read real quotes from published authors. 